\title{
Localization of topological changes in Couette and Poiseuille flows of two-dimensional foams
}

\author{
S.J. Cox and A. Wyn \\ Institute of Mathematics and Physics, Aberystwyth University, SY23 3BZ, UK
}

\begin{abstract}
Quasistatic simulations show that the topological changes or plastic events that occur when an aqueous foam flows are confined within narrow regions. The width of these regions is shown to scale with a power of the polydispersity in bubble area. The exponent is one-half for linear Couette flow and one-fifth for Poiseuille flow.
\end{abstract}

Keywords: Foams, Rheology, Localization, Plasticity

PACS: $47.57 . B c$ Foams and Emulsions, 83.80.Iz Emulsions and Foams

\section{INTRODUCTION}

Liquid foams are familiar from domestic use and important in industrial applications, including ore-separation and enhanced oil recovery. They are elasto-visco-plastic complex fluids with a highly nonlinear response to applied forces: at low strain they deform elastically, like a solid, while above a yield stress they flow like a viscous liquid. Of all complex fluids, liquid foams provide one of the most experimentally accessible systems for study, since bubbles are objects that can have millimetric dimensions. Moreover, Plateau's laws mean that the internal structure of a foam is well understood, at least at the level of the network of films [1]. Foams thus provide a prototypical complex fluid.

We study two-dimensional (2D) foams, such as can be made by squeezing a foam between parallel glass plates until it consists of a single layer of bubbles. Despite its simplicity in comparison to the three-dimensional foams found in applications, the dynamics of 2D foams are rich, and provide insight into their 3D counterparts.

The mathematical idealization of a two-dimensional foam is as follows: a dry 2D foam at equilibrium consists of bubbles with fixed areas surrounded by films that are circular arcs meeting threefold at angles of $120^{\circ}$. These rules are consequences of minimization of energy [2], which is in this case the total film length multiplied by surface tension. We use the Surface Evolver software [3] to simulate, with high accuracy, foams consisting of many hundreds of bubbles in order to predict their plastic response.

At low strain a foam responds as an elastic medium. As the strain increases, the foam begins to yield and bubbles begin to slide past each other in plastic events known as $T_{1}$ topological changes [1]. These occur when a film shrinks to zero length and a fourfold vertex is formed. Such a vertex is unstable, and immediately dissociates into two threefold vertices with the connecting film now perpendicular to the vanishing one.

Localization of $\mathrm{T}_{1}$ events, also referred to as shear banding, has been described in experiments in an annular widegap Couette viscometer [4]. After an initial transient, the majority of $\mathrm{T}_{1}$ events occur close to the inner moving wall. Similar results have been found in simulations [5]. In linear Couette shear between parallel side-walls, there is also localization of $\mathrm{T}_{1} \mathrm{~s}$. In this geometry, since the shear stress should be homogeneous there is no preferred location for the localized region based upon the boundary conditions, confirmed by Potts model [6] and Surface Evolver [7, 8] simulations. However, strain ordering close to the boundary means that the localization does often occur there.

Previously, we predicted that the width of the localized region in linear Couette shear depends strongly on the area disorder of the foam [8]. This is, in effect, a prediction of the degree to which the foam is fluidized under shear. In the limit of zero area disorder - a monodisperse foam - $\mathrm{T}_{1}$ events tend to occur in a very narrow band and shearinduced crystallization is evident. Making a foam more polydisperse widens the localized region and can thus reduce the amount of static foam present.

We characterize the polydispersity, or volumetric disorder, of a foam by the second moment of the distribution of bubble areas $A$ :

$$
\mu_{2}(A)=\left\langle\frac{(A-\langle A\rangle)^{2}}{\langle A\rangle^{2}}\right\rangle
$$

where \langle\rangle denotes an average over the whole foam. 
(a)

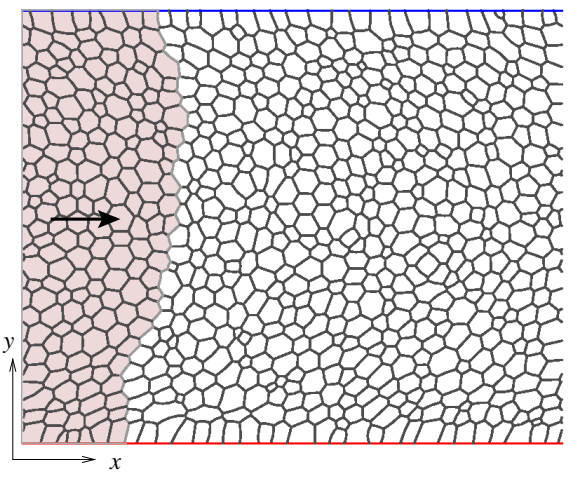

(b)

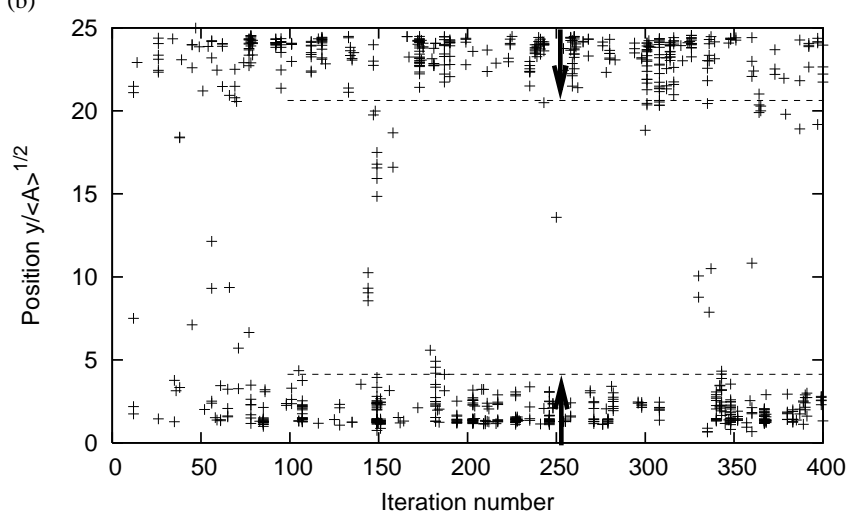

FIGURE 1. (a) An example of the foams used to simulate Poiseuille flow of a 2D foam for $\mu_{2}(A)=0.123$. (b) The $y$ positions of the $\mathrm{T}_{1}$ s during flow for $\mu_{2}(A)=0.170$.

In quasi-static simulations of linear Couette shear (force-driven flow), we found that the width $w_{l}$ of the localized region, normalized by the bubble size, is proportional to the square-root of area disorder [8]:

$$
\frac{w_{l}}{\sqrt{\langle A\rangle}} \approx \mu_{2}(A)^{\alpha}, \quad \alpha=0.489 \pm 0.0634
$$

Here, we investigate whether a similar dependence on $\mu_{2}(A)$ is found for Poiseuille (pressure-driven) flow. That is, we consider a channel with a no-slip boundary condition and push the foam along the channel. Topological changes occur as the bubbles slide past those fixed to the walls, and we ask how the width of the regions in which $\mathrm{T}_{1} \mathrm{~s}$ occur varies with the area dispersity of the foam.

\section{NUMERICAL METHOD}

We use the Surface Evolver [3] in a mode in which each film is represented as a circular arc. A typical foam is shown in fig. 1(a): it consists of $N=738$ bubbles confined between parallel walls $[8,9]$ a distance $W=0.804$ apart, in a channel of unit length. The value of surface tension, which should be thought of as a line tension with units of energy per unit length, is taken equal to one throughout. In contrast to the disorder in the number of sides $n$ of each bubble, $\mu_{2}(n)=\left\langle(n-6)^{2}\right\rangle$, which varies in time due to $\mathrm{T}_{1} \mathrm{~s}$, the area disorder is fixed in each of our simulations; that is, we exclude inter-bubble gas diffusion (coarsening) and film collapse.

The simulation procedure is as follows. A Voronoi construction [10] is first used to generate a fully periodic tessellation of the plane. Bubbles at the top and bottom are sequentially deleted until the required number remains. This structure is imported into the Surface Evolver and new bubble areas are determined randomly from a Weibull distribution [8]. The channel is thus periodic in the $x$ direction and films that meet the side-walls have their ends pinned to the wall (no-slip condition). A realistic foam structure is found by minimizing the total film length subject to the prescribed bubble areas. During this minimization $\mathrm{T}_{1} \mathrm{~s}$ are triggered by deleting each film that shrinks below a certain length $l_{c}$ and allowing a new film to form. The critical length $l_{c}$ is a measure of liquid fraction $\Phi[9,11]$, but we keep it small enough here $\left(l_{c} \leq 0.005\right.$ throughout, corresponding to $\left.\Phi \leq 10^{-4}\right)$ that it should not affect the results.

Flow is initiated by choosing at random a line of films connecting the two walls, defining a region of the foam (shown shaded in figure 1(a)). At each step the area of this region is enlarged in small increments $d A_{0}=0.001$; the precise motion that ensues is a further variable in the minimization procedure [9].

\section{RESULTS}

For each value of area disorder, we record the $(x, y)$ position of each $\mathrm{T}_{1}$ and the iteration number at which it happens. As expected, many $\mathrm{T}_{1} \mathrm{~s}$ occur around one bubble width from each wall - see fig. 1(b). We simulate the flow for 400 


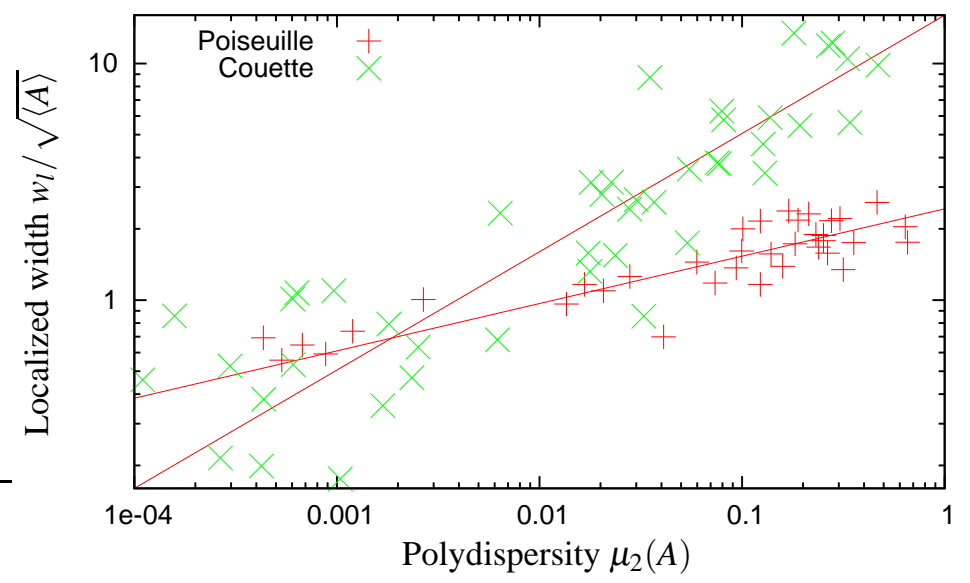

FIGURE 2. Localized width as a function of polydispersity. The lines have slopes of one-half and one-fifth.

iterations, corresponding to an equivalent $x$-displacement equal to half the length of the channel (but since not all bubbles move, a bubble close to the centre of the channel will move farther than this). We discard data from the first 100 iterations, to eliminate any transient that might arise from any artificiality in the initial structure, and count the number of $\mathrm{T}_{1} \mathrm{~s}$ that are found in the upper and lower halves of the channel. Then we calculate the distance $w_{\text {lower }}$ from the lower wall within which $90 \%$ of the T1s in the lower half of the channel occur, and do the same for the upper half, marked with arrows and dashed lines on fig. 1(b). We combine these and subtract the diameter of the bubbles next to the wall to find the localized width:

$$
w_{l}=\frac{1}{2}\left(w_{\text {lower }}+\left(W-w_{\text {upper }}\right)\right)-\sqrt{\frac{4\langle A\rangle}{\pi}}
$$

Figure 2 shows the localized width $w_{l} / \sqrt{\langle A\rangle}$ for both linear Couette [8] and Poiseuille flows. The power-law relationship between width and area dispersity is robust, and in this respect Couette and Poiseuille flows are similar. However, the exponent is much less in the Poiseuille case - a fit gives $\alpha=0.180 \pm 0.028$ - indicating that in pressuredriven flows such as this, in which the localization is confined to the walls, the localized width grows rather slowly and polydispersity plays a lesser role in the flow dynamics.

\section{ACKNOWLEDGMENTS}

We thank K. Brakke for his development and maintenance of the Surface Evolver code and acknowledge financial support from EPSRC (EP/D048397/1, EP/D071127/1).

\section{REFERENCES}

1. D. Weaire, and S. Hutzler, The Physics of Foams, Clarendon Press, Oxford, 1999.

2. J. Taylor, Ann. Math. 103, 489-539 (1976).

3. K. Brakke, Exp. Math. 1, 141-165 (1992).

4. G. Debrégeas, H. Tabuteau, and J. di Meglio, Phys. Rev. Lett. 87, 178305 (2001).

5. S. Cox, Simulations of Two-Dimensional Foam under Couette Shear (preprint) (2007), http://hdl.handle.net/2160/323.

6. Y. Jiang, P. Swart, A. Saxena, M. Asipauskas, and J. Glazier, Phys. Rev. E 59, 5819-5832 (1999).

7. S. Cox, D. Weaire, and J. Glazier, Rheol. Acta 43, 442-448 (2004).

8. A. Wyn, I. Davies, and S. Cox, Euro. Phys. J. E in press (2008).

9. S. Cox, B. Dollet, and F. Graner, Rheol. Acta. 45, 403-410 (2006).

10. K. Brakke, 200,000,000 Random Voronoi Polygons, www.susqu.edu/brakke/papers/voronoi.htm (1986), Unpublished.

11. C. Raufaste, B. Dollet, S. Cox, Y. Jiang, and F. Graner, Euro. Phys. J. E 23, 217-228 (2007). 